Bikas S. Aghayev

DOI: $10.25045 /$ jpit.v08.i2.11

Institute of Information Technology of ANAS, Baku, Azerbaijan

depart6@iit.ab.az

\title{
CLASSIFICATION OF THE INTEGRATED INFORMATION SYSTEMS IN LIBRARIES
}

The article deals with the integrated information systems in libraries. It explores the impact of the automation on the efficiency of the library's performance and the expansion of its scope. Furthermore, it offers some recommendations for increasing the efficiency of the electronic libraries.

Keywords: library, electronic library, library operations, information systems, e-catalog, e-library funds.

\section{Introduction}

The process of evolution of the civilization has stepped into a new era of information -the digital era, leaving behind the global information revolutions related to the emergence of the speech and writing, the invention of the writing machine, telegraph, radio and television. This era is characterized by the emergence of a global electronic information environment arising from the unity of computer and telecommunication technologies. From the emergence of the publication especially of books, libraries have been the only entity systematically and consistently collecting, systematizing, storing and delivering manuscripts, printings, art and other material and spiritual works to the users [1]. Indeed, only a library has been a place providing the use of professionally selected and classified information that is the knowledge generated by the humanity throughout the history. In other words, during this period, the library has played the role of an intellectual mediator and coordinator between the society and the media. Due to the level of development of the techniques and technology, the library work was limited to access, storage, and use of documents, rather than the information they carry, so that this activity could satisfy only a limited number of users. In the information society characterized by the information abundance generated by the information and communication technologies (ICT), the classical libraries working with paper carriers do not meet the requirements of the day and are compressed from the information market by more advanced, multifunctional, relevant and convenient libraries. This is primarily due to the disadvantages of the paperwork, which is the main object of the classical library: these documents do not include audio, video, animation, multimedia data, and their production, circulation and recovery are complicated and expensive, and the distribution is slow and perishable [2, 3]. Electronic publications and digital media are free from all these deficiencies, requiring a compact storage space, and their correction and circulation do not require additional paper or printing expenditures being more operative and simple, moreover, the database search and access are easy and convenient. Therefore, to preserve the importance as a social information institution, the libraries should be able to provide the users with information in all available forms created by the humanity. This is possible only by creating new types of libraries - electronic libraries (EL), that meet modern requirements through the automated library work based on the latest techniques and technologies.

The article examines the integrated information systems of libraries, and provides some recommendations to improve the efficiency of electronic libraries.

\section{Automation of library-bibliographic work}

As mentioned in the introduction, insufficiently automated library work does not meet up to date standards. Here, the "automation" means the creation of a digital library fund, the application of the automated library information system (ALIS), as well as the automation of many processes that directly affect the performance efficiency of the library rather than the library activity. In other words, automation may cover the direct library-bibliographic activities, including some non-core processes, such as different types of services, security and protection. Evidently, the main functions of the traditional or the EC are: 
- building the library fund;

- organizing convenient search of the resources from the library fund to the user (reader);

- fast and convenient delivery of the search results to the user.

EL performs these tasks in a more convenient and convenient manner for the user by using modern technologies, especially ICT capabilities: the user searches for the resource in electronic catalogs instead of the usual paper catalogs, and the found original material or its copy is delivered to the reader typically or through e-mails over a computer/telephone network. In any case, the main purposes of the automation are $[4,5]$ :

- Increasing the effectiveness of the library work;

- Improving the quality of the provided products (information) and services;

- Facilitating the work of the library staff.

These goals are achieved through the use of appropriate techniques and technologies, software and organizational tools. Obviously, in order to achieve these goals, first of all, the above-mentioned key areas of library activity should be automated. The transition from the status of the "traditionalism" to "electronic library" is also determined primarily by the automation of these operations. Because, according to the generally accepted definition, EL is characterized as an information system collected in the databases of the managed electronic (digital) documents. For this purpose, various software packages called "Automated Library Information Systems" (ALIS) are used.

The goal of using these information systems is to automate the following major library activities:

- creating electronic catalog database, providing of convenient navigation and search tools;

- creating bibliographic library fund - databases in accordance with the profile of the library (minimally problematic or better summarized).

The ALIS is a software-hardware set, consisting of software modules that automate a certain number of library functions, automated workstations and maintenance tools. At present, there are a large number of SARS, which differ according to the factors, such as designation, functional capabilities, price, maintenance characteristics, etc. Some of the widely used systems are mentioned below.

"VIRTUA". This system (USA, VTLS company) is designed to generally automate the library work. Marc 21-based e-catalog (meta-database) with extensive search capabilities has numerous functions such as forming different profile funds, inter-library exchange (according to Z39.50 protocol), and statistical data processing and so on.

It has client-server architectural network capabilities (library services over local and global networks), including a convenient Web interface for remote users, supports UNICODE national characters, and uses the Oracle database management system (DBIS) to archive contents, such as e-catalogs, e-documents and etc. It is mainly used in large libraries (national, academic, general use, etc.).

"ALEPH". However, this ALIS (Israel, Exlibris Company) has less functionality than the "VIRTUA", it also enables the automation of basic library operations. All Israeli libraries centrally use only this system [6]. ALEPH can operate within the local network and the Internet: it is open system and all library resources are accessible with the standard procedures of the Internet technology. Its search format, VBIS, font format, ACSI exchange protocol, and etc. are the same as in VIRTUA. It is mainly intended for medium-sized specialized and corporate libraries.

“LIBER"(France, Relais Informatique International), IRBIS (Integrated Library Information System, Moscow, Russia), ALEPHINO (Exlibris Company) and other ALISs are also widely used and each has its own functionality and destination indicators.

The library is considered to be the most perfect area for information structuring and classification. This is primarily due to the fact that the automation of library information with digital content is more favorable: the development of the electronic catalogs and bibliographic fund, which is the main object of the library, is realized by the method of the digitalization of 
primary analogue materials. The digitalization is the most effective way to preserve the publications, art samples, provide access to digital copies included in the databases and implement certain operations on them. Any ALIS is a system that incorporates and manages the e-format catalogs and fund materials in the databases by digitizing.

The automation of the digitalization is carried out in two ways: scanning and photocopying (with camera). The method of the selection is determined by the properties of the description object to be digitized. The photocopying method, as a rule, is used for the digitalization of voluminous stock materials (for example, museum exhibits), large-sized surface materials (pictures, decorations), and the materials that cannot be brought into the workplace with scanning equipment. The scanning is more convenient for digitizing the printed materials (printings and handwritings). As the photocopying clearly and accurately reflects the texture of the material - smaller parts, it generates a higher quality electronic document than the scanning. However, unlike scanning, the efficiency of photography is very low: depending on the object's texture, its dimensions, the brightness of its surface, and other factors, selection of the angle and lighting mode for each object is time consuming.

The digitalization settings are defined according to the relevant standards: 300 dpi (higher for photocopy), file format TIFF or JPEG, dimensions - 130x80 for catalogs text, as required for other objects (print materials, descriptive artwork, etc.). The correction work to improve the quality of digitized documents is also automated using software tools: the purpose is to ensure the authenticity of the original document with the electronic one. Formation of databases from the electronic document massifs (retro-conversion operation) is carried out automatically within the ALIS capabilities. Document formatting (MARC, RUSMARC, etc.) is determined by the system's description. When selecting the digitalization software, the features of the automation object must be specified. For example, the automation software may vary depending on the catalogue types (alphabetical catalogue, system catalogue, service catalogue, reader directory etc), original (machine printing, handwriting), required quality of the e-copy (polygraphic quality - long-term storage, user-quality, service quality), retro-conversion objectives and so on.

For the automation of the scanning operations high-quality professional cameras, document scanners (for page-type documents - contact method) and planetary scanners (for large objects distant description) are used (Fig.1).

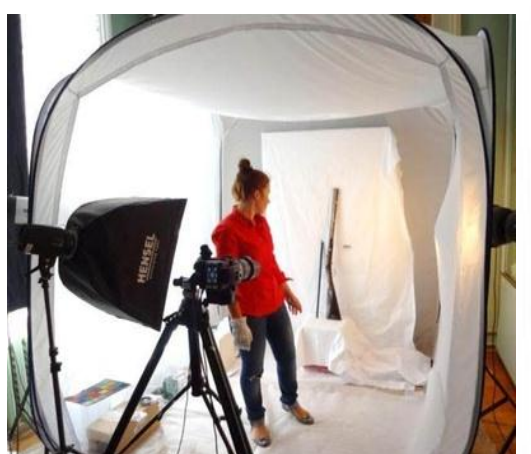

Photographing

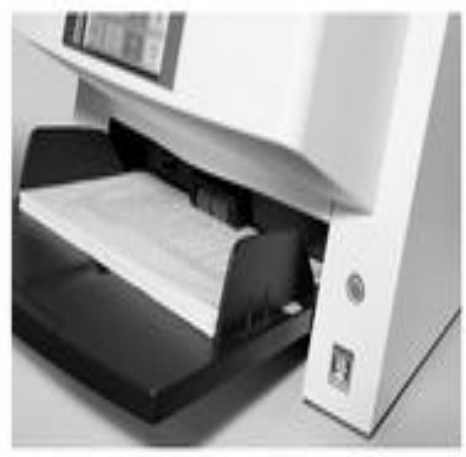

Document scanner

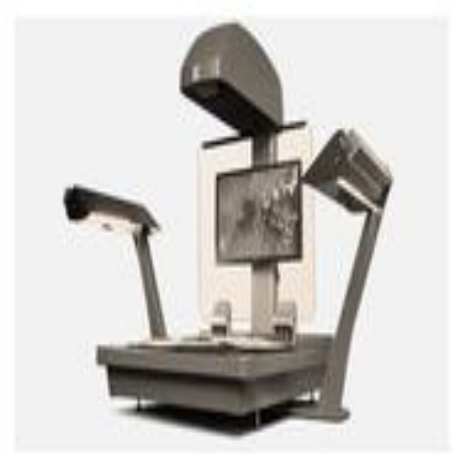

Planetary scanner

Figure 1. The digitalization equipment samples

Nowadays, different advanced digitalization technologies and methods have been developed to generate the digital images of either printed materials or objects. Using these tools, a wide range of general-purpose modern libraries are expanding their service scope and user base by digitizing the audiovisual, photo, cinema, animation, fine arts and other materials and uploading them into the appropriate media sub-bases for art works, photos, cinema, and etc. Data-mining, cloud technologies, mobile versions of the Web sites, and other perspective techniques and technologies are also the factors that enhance the automation of the library work [7]. 


\section{Other automation tools used in the library}

As mentioned above, in modern libraries, besides bibliographic activities, a number of other related processes are automated. These automation tools can be grouped into the following groups for their functions [8]:

- Communication tools. This group includes mainly internal and urban telephone network, dispatcher communication system, voice notification system, teletext, videotext, corporate computer network, Internet, videoconferencing system, and etc. The wireless dispatcher communication system within the library enables operative and effective management of the moving staff (especially in large libraries);

- Individual and collective audiovisual tools. These tools can be used to conduct scientific, cultural, and other events. This group includes audio-video players, dictaphones, audio and video recorders, film cameras, radio-television equipment, cameras, projectors, etc;

- Informational tools. This group includes various types of individual and collective tables, screens, and so on;

- Microfilming tools. The automation of the microfilming allows building the microfilm fund of the library (microcopy fund), which meets the need of additional readers' group.

- Security systems. These systems may include:

- video surveillance system;

- fire safety system;

- alarm system for the theft of library resources, etc.

Video surveillance system (VSS) performs the functions of controlling the inadequate movement of readers and library personnel and the detection of unauthorized access to the library site and the abduction of library resources. VSS consists of the cameras installed along the perimeter of the library, its corridors, stock area, including the computer hardware (server equipment) that receives, processes and archives the video shots, and the monitors at the operator's workplace. The operator observes the events on the monitor and takes adequate measures when detecting unusual incidents. It is recommended to use HD quality cameras with high resolution to make clearer images (events), for example, to recognize the human face. To avoid continuously watching the monitor, some modern VSSs are equipped with intelligent analysis software to automate the incident detection [9] (Figure 2).

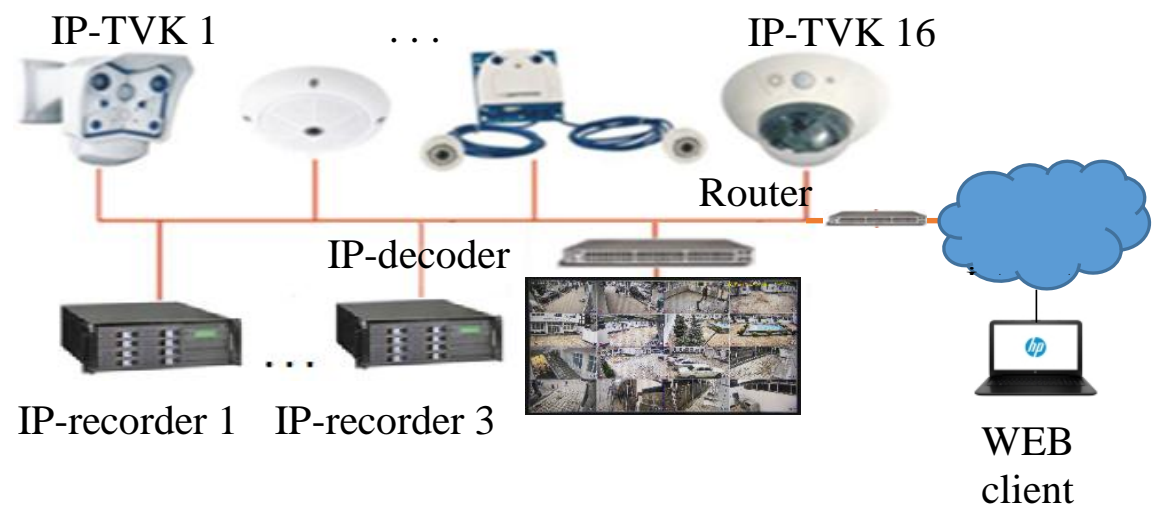

Figure 2. Video surveillance system samples

Fire safety system. It detects smoke, high temperatures and fire in the library area and generates noise and warning signals in these cases. More advanced systems are integrated with VSS, displaying the location of the fire and the incident at the operator's monitor and launching fire-fighting devices [10].

Anti-theft alarm system. The abovementioned VSS partially performs this function. However, the incidents may occur beyond the scope of the video cameras, thus the description of 
the attacker's face and other features may not be clear. Therefore, it is more appropriate to use special tools, such as, Radio Frequency Identification (RFID) (Figure 3).

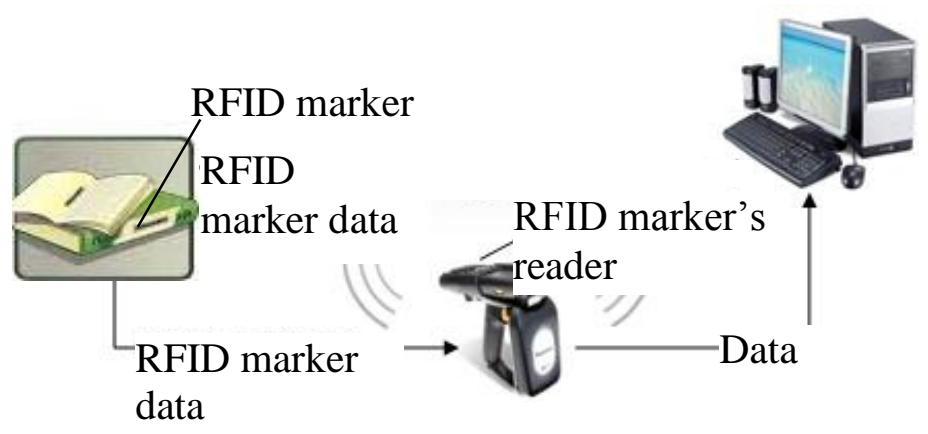

Figure 3. Architectural scheme of RFID system

RFID technology is mainly used to automate the following processes:

- identifying the theft of books and magazines;

- delivering(recording) and receiving (removing) the publications to/from the reader;

- publications search and inventory.

RFID technology is based on the remote reading and identification of the code (RFID marker) written on the memory of the micro-scheme by the receiver (scanners). Markers or ordinary barcodes are attached to the books and other materials of the stock. The RFID code of the book taken by the reader is automatically activated in its electronic reader booklet and in the memory of electronic device installed at the exit. At the exit, the scanner "reads" the code in the book and compares it to the others stored in the cache. When the codes differ, the incident is identified as a theft and a warning alert activates, which identifies the reader's ID according to the RFID code [11]. The RFID system can be integrated with VSS to increase the accuracy of the recognition process.

The automation of the mentioned operations is based on the automated identification, activation/deletion of the location of the requested book in the reader's directory and database and the facilitation of the sorting process during the inventorying(by avoiding the librarian to read the book title) by the RFID scanner. Advanced ALISs comply with the hardware-software tools of RFID equipment.

Tiflology (tiflo-technics). The services delivered to the blind and visually impaired people are the other areas of the library which are automated. Over the past decade, a number of simple techniques and technologies have been developed to provide library services to the people with disabilities. Utilizing these tools, they can use resources from the library stock as well as the resources from the other libraries being provided with the access to the Internet from the reader's place equipped with special equipment. To this end, the computer of these people is equipped with Braille devices (display, keyboard, printer, etc.) and text-speech synthesizer. It authorizes the library stock materials and downloads them to the computer and sounds them in "reading book" mode. Currently, many libraries perform the automation work mainly by two ways to provide the disabled people with the library services [12]:

- building a Braille fund consisting of dots for blind and visually impaired people in the library and providing the readers' desktop with Braille computer equipment;

- establishing an audio fund of "speaking books"- audio version of ordinary printed materials recorded on the electronic media. For example, INFA branded automated universal reader station based on text-speech software enables the reader to choose material from the electronic catalog without referring to the librarian, to download it to the station, to read, and remove from his/her card(to return the book) [13]. 
The organization of the work of the Electronic Library Center of the Institute of Information Technology of ANAS

The library of the institute has established EL based on Alephino (Israeli, German branch of Exlibris) ALIS database. The system has been developed on the AzScienceNet science network of ANAS, and it is one of the network services provided to the users [14]. The foundation works include the publications of the Institute's staff, a number of books and periodicals on the Institute's profile, abstracts and dissertations. The works of the Institute employees, including the texts of dissertations and their abstracts are submitted to the library as electronic texts. The abstracts and other printed materials of other employees of ANAS are digitized by scanning method and included into the EL Fund.

Besides the library services, the librarians also perform research, statistics and analytics.

The following processes of the institute's library are automated:

- preparing the electronic readers' cards (brochures);

- summarized electronic catalog (mainly based on the scientific works bythe Institute's staff and the abstracts of the dissertations by the employees ofANAS);

- authorized database access;

- archiving of the proceeding of the conference conducted at the institute in ALIS;

- digitization of the orders and requests;

- deleting the returned material at self-check station installed in the reading hall;

- detecting the theft (through RFID technology);

- registering(with the indication of the user, number of accesses, article titles and etc.) and statistical processing the accesses and orders;

- notifying about the library fund, accesses, services, rules of use, etc. online.

Despite the fact that the library is a narrow and corporate area, it should expand its automation to improve the quantitative and qualitative indicators of the services, especially electronic services. Therefore, the study offers the following measures to increase the efficiency of the library:

1. Transiting from corporate to public libraries, and commercializing the e-services.

2. Increasing the range of full-text electronic materials. First of all, submitting the works by the institute's staff to the database (with their copyright).

3. Integrating the institute's audio-video galleries into the electronic library fund.

4. Installing VSS at reading hall, stock and other areas of the library and integrating with the RFID anti-theft device.

5. Applying the fire-safety alarm system.

6. Providing convenient and reliable network access to the e-library for the institute departments beyond the library area.

\section{Conclusion}

As a result of the case study, it was concluded that the library is the only public and social institution, in which the works have been collected, systematized and used since the emergence of writing until the digital age. However, new types of electronic libraries, based on recent achievements in techniques and technology, have lessened the significance of the classic libraries and shrunk them. This article explored the essence of this phenomenon. It showed that the library should not only focus its work on paper carriers, but should also work with all forms of information by forming digital information environment with art works, photo gallery, izoteka, cinema gallery and media to preserve the status of social institution as the mediator between the people and information and knowledge. Therefore, it is justified that the automation is the most important tool to achieve this goal. The article also explored the automation of library activity, management and security through the use of digitalization methods, communication systems and the Internet, individual and collective use of audiovisual and informational tools, RFID and others. The 
automation of the work at the Electronic Library Center at IIT of ANAS was surveyed and recommendations were put forward to increase the effectiveness of the work.

\section{References}

1. Lukanova E.A. The use of information and communication technologies in the college library. elibrary.udsu.ru/xmlui/bitstream/123456789/6243/Использованиеинформационнокоммуникационныхтехнологий в библиотеке колледжа.pdf? Sequence $=1$

2. Automation of library activities. Www.allrefs.net/c99/1g1lw/

3. The use of multimedia technologies in library. Information-methodical digests. Novosibirsk: Pub.house NSRSL, 2012, 68 p. www.ngonb.ru/docs/ Methodists / Multimedia.pdf

4. Lobuzina E.V. Electronic resources of the scientific library in the modern information environment // Information support of science: new technologies. www.benran.ru/SEM/Sb_11/sbornik/doc_344.pdf

5. Libraries and information resources in the modern world of science, culture, education and business. http://gpntb.ru/win/inter-events/crimea2015/

6. Software ALIS. http://laleshin.narod.ru/pto/T-4-2.pdf

7. Alguliyev R., Ismayilova N. Perspectives of the future generation electronic libraries / Republican scientific-practical conference "Problems of formation of e-libraries", Baku, 2016, pp. 8-12.

8. Aleshin L.I. Software and hardware for automated library and information systems. Training and methodologybook. MSUCA, 2010. http://laleshin.narod.ru/PTO-ABIS_UMK.pdf

9. Aghayev B.S. About the challenges of processing of large-scale video information resources / Proceedingsof the First republic scientific-practical conference "Big data: opportunities, multidisciplinary problems and perspectives", Baku, 2016, pp. 49-53.

10. Anti-theft systems for libraries. www.armosystems.ru/system/3m_library.ahtm

11. Operation principle of RFID technology and its application. https://rtlservice.com/ru/company/blog/princip_raboty_tehnologii_rfid_i_ee_primenenie/

12. The Tuva Republican Special Library for the Blind and Visually Impaired. http://tifloknigatuva.ru/content/sprashivayte-otvechaem

13. Kravtsova O.A. Electronic libraries: state-of-the-art and development prospects, Kharkov KSAA, 2013. http://en.calameo.com/read/00284123724acc192ab08

14. Alakbarov R., Mustafayev T., Yagubov M. Electronic library service in AzScienceNetscience computer network / Republican scientific-practical conference "Problems of e-libraries formation”, Baku, 2016, pp. 99-101. 